\title{
Lie Group Methods for Eigenvalue Function
}

\section{Nazarkandi HA*}

Departement of Mathematics, Marand Branch, Islamic Azad University, Marand, Iran

\begin{abstract}
By considering a $C^{\infty}$ structure on the ordered non-increasing of elements of $R^{n}$, we show that it is a differentiable manifold. By using of Lie groups, we show that eigenvalue function is a submersion. This fact is used to prove some results. These results is applied to prove a few facts about spectral manifolds and spectral functions. Orthogonal matrices act on the real symmetric matrices as a Lie transformation group. This fact, also, is used to prove the results.
\end{abstract}

Keywords: Lie group; Spectral manifold; Submersion; Eigenvalue function; Spectral function

\section{Preliminary}

Let's $S(n, R)$ be the space of real symmetric matrices and $O(n, R)$ the group of all real orthogonal matrices. For any $A \in S(n, R)$ its (repeated) eigenvalues $\lambda_{1} \geq \ldots \geq \lambda_{n}$ are real, and it admits a spectral decomposition of the form

$$
A=\operatorname{Pdiag}\left[\lambda_{1}, \ldots, \lambda_{n}\right] P^{t}, \quad(1.0)
$$

(Where $\mathrm{A}$ is a symmetric matrix, $\mathrm{P}$ is an orthogonal matrix $(\mathrm{PP} \wedge \mathrm{t}=\mathrm{I})$, lambda_1,... llambda_n are eigenvalues of $\mathrm{A}$.)

for some $P \in O(n, R)$, where $\operatorname{diag}\left[\lambda_{1}, \ldots, \lambda_{n}\right]$ is the diagonal matrix with its ith diagonal entry $\lambda_{i}$ and $P^{t}$ is the transpose of matrix $P$. Note that (1.0) is independent of the choice of $P \in O(n, R)[1,2]$.

Let $\lambda():. S(n, R) \rightarrow R^{n}$ be the eigenvalue function such that $\lambda_{i}(A), i=1, \ldots$ ,$n$, yield eigenvalues of $A$ for any $A \in S(n, R)$ and are ordered in a nonincreasing order, that is, $\lambda_{1}(A) \geq \ldots \geq \lambda_{n}(A)$. For applications of this function in variational analysis of spectral functions, semidefinite programs, engineering problems, nonsmooth analysis and at least in quantum mechanics [3-7].

We recall that, matrices $A, B \in R^{n \times n}$ are similar, if $B=S^{-1} A S$ with $S \in$ $R^{n \times n}$ invertible.

If $B$ similar to $A$, then $B$ and $A$ have the same eigenvalues.

In the following, we reviewed some the formal definitions, theorems and examples of differentiable manifolds [8]. Also any book on the theory of differentiable manifolds may be used for reference if necessary.

\section{Definition 1.1}

1. A diffeomorphism $f: R^{n} \rightarrow R^{n}$ is an injection such that both $f$ and its inverse $f^{-1}$ are $C^{\infty}$ function.

2. Let $M, M^{\prime}$ are two differentiable manifolds, $\varphi: M \rightarrow M^{\prime}$ be a mappi $M \mathrm{ng}, m \in M, \varphi m \in M^{\prime} . \varphi$ is called a submersion if its rank (rank of it's Jacobian matrix) is equal to the dimension of $M^{\prime}$ at each point of its domain. $\varphi$ is called an immersion if its rank is equal to the dimension of $M$ at each point of its domain.

3. A manifold $M^{\prime}$ is said to be a submanifold of a manifold $M$ if it is a subset of $M$ and if the natural injection $j: M^{\prime} \rightarrow M$ is an immersion.

4. Let $\varphi: M \rightarrow M^{\prime}$ be any function. A section of $\varphi$ is a function $\psi$ : $M^{\prime} \rightarrow M$ such that $\varphi_{\circ} \psi$ is the identity function on the domain of $\psi$.
For example, the set $S(n, R)$ of real symmetric $n \times n$ matrices is a submanifold (with dimension $n(n+1) / 2$ ) of the set of all real matrices $M(n \times n, R)$.

When the topology on $M^{\prime}$ induced by its $C^{\infty}$ structure is its topology as a subset of $M, M^{\prime}$ is said to be a regular submanifold of $M$.

Next proposition is about submersions and it plays key role in the differentiability of eigenvalue function [8], propositions 6.1.2; 6.1.4; 6.2.1).

\section{Proposition 1.1}

1. $\varphi$ is a submersion of $M$ onto $M^{\prime}$. If $\psi: M^{\prime} \rightarrow M^{\prime \prime}$ is such that $\psi \circ \varphi$ is differentiable then $\psi$ also is differentiable.

2. A differentiable function $\varphi: M \rightarrow M^{\prime}$ is a submersion if and only if for each $m$ in its domain there exists a differentiable section of $\varphi$ containing $m$ in its range.

3. Let $\varphi: M \rightarrow M^{\prime}$ is a submersion. If $\operatorname{dim}(M)>\operatorname{dim}\left(M^{\prime}\right)$, each set $\varphi^{-1}(s)$ has the structure of a regular submanifold of $M$ of dimension $\operatorname{dim}(M)-\operatorname{dim}\left(M^{\prime}\right)$.

\section{Definition 1.2}

1. A Lie group $G$ is a group which has the structure of a differentiable manifold and for which the group function

$$
\theta: G \times G \rightarrow G
$$

defined by $\left(g_{1}, g_{2}\right) \rightarrow g_{1} g_{2}$ is differentiable.

2. A Lie group is said to act on a differentiable manifold $M$ as a Lie transformation group if we are given a global surjection

$\Phi: G \times M \rightarrow M$

which is differentiable and such that if $g, h \in G$ and $m \in M$

$\Phi(g, \Phi(h, m))=\Phi(g h, m)$.

If $g \in G$, the function $\Phi: M \rightarrow M$ defined by $m \rightarrow \Phi(g, m)$ is a diffeomorphism of $M$ onto itself.

*Corresponding author: Nazarkandi HA, Departement of Mathematics, Marand Branch, Islamic Azad University, Marand, Iran, E-mail: halizadeh@marandiau.ac.ir

Received February 13, 2016; Accepted June 15, 2016; Published June 20, 2016

Citation: Nazarkandi HA (2016) Lie Group Methods for Eigenvalue Function. J Generalized Lie Theory Appl 10: 240. doi:10.4172/1736-4337.1000240

Copyright: @ 2016 Nazarkandi HA. This is an open-access article distributed under the terms of the Creative Commons Attribution License, which permits unrestricted use, distribution, and reproduction in any medium, provided the original author and source are credited. 
Let $G L(n, R)$ be non-singular real matrices. The set $O(n, R)$ of real orthogonal $n \times n$ matrices can be given the structure of a Lie subgroup of $G L(n, R)$ of dimension $\frac{1}{2} n(n-1)$. This Lie subgroup is a regular submanifold.

$G L(n, R)$ acts on $R^{n}$ as a Lie transformation group with the function $\Phi$ defined by $(A, z) \rightarrow A z$.

Also, $O(n, R)$ acts on $S^{n-1}$, unit sphere of $R^{n}$, as a Lie transformation group. This action is transitive.

\section{Differentiability of $\lambda($.}

In this section our aim is to prove that $\lambda$ is a differentiable function between two differentiable manifolds. For this work, we will consider a $C^{\infty}$ structure on the ordered non-increasing of elements of $R^{n}$. We start with a remark:

\section{Remark 2.1}

1. Let $f: A \rightarrow M$ be a bijection on a set $A$ with values on a differentiable manifold $M$. Then $f$ defines a complete $C^{\infty}$ atlas of $A$. With this structure on $A, f$ is a diffeomorphism [8].

2. Let $n \in N$ (natural numbers)is a finite cardinal number and $c$ be the cardinal of real number of $R$ [9]. We recall that

(a) $n+c=c$

(b) $c+c=c$

(c) For each $A, B$ sets

$\operatorname{card} A+\operatorname{card} B=\operatorname{card}(A \cup B)+\operatorname{card}(A \cap B)$.

Where A, B are arbitrarily sets, cardA and cardB are their cardinal numbers.

Denote by $R_{z}^{n}$ the ordered non-increasing of elements of $R^{n}$. That is,

$R_{\geq}^{n}=\left\{\left(x_{1}, \ldots, x_{n}\right) \in R^{n} \mid x_{1} \geq \ldots \geq x_{n}\right\}$.

It is clear that $R_{\geq}^{n}$ is a convex, closed subset of $R^{n}$. The next result show that $R_{\geq}^{n}$ is bijective with $R^{n}$.

\section{Lemma 2.1: Let}

$R_{\geq}^{n}=\left\{\left(x_{1}, \ldots, x_{n}\right) \in R^{n} \mid x_{1} \geq \ldots \geq x_{n}\right\}$.

Then $R^{n}$ is bijective with $R_{>}^{n}$.

Proof 2: Let $x=\left(x_{1}, \ldots, x_{n}\right) \in R^{n}$. There are $n !$ (factorial)possible orders for $x$ components. So there are $n$ ! permutation matrices as $P_{1}, \ldots, P_{n !}$ gives rise to these orders in matrix notation. If $P_{0}$ shows identity matrix and $P_{i} R_{\geq}^{n}=\left\{P_{i} s \mid s \in R_{\geq}^{n}\right\}, i=0, \ldots, n$ ! then we have:

$R^{n}=P_{0} R_{\geq}^{n} \cup P_{1} R_{\geq}^{n} \cup \ldots \cup P_{n !} R_{\geq}^{n}$.

(Where $P \_i$ is a permutation, $R^{\wedge} n$ is the set of $n$-tple of ordered real numbers, $\mathrm{C}$ is cardinal of real number set)

But $P_{i} R_{\geq}^{n}$, is bijective with $R_{\geq}^{n}$. So $\operatorname{card}\left(P_{i} R_{\geq}^{n}\right)=\operatorname{card}\left(R_{\geq}^{n}\right)=c$. By the above remark, part 2

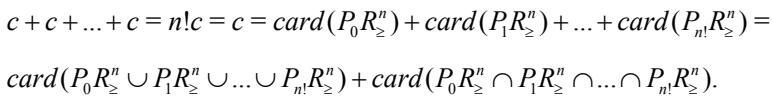

But

$k=\operatorname{card}\left(R_{\geq}^{n} \cap P_{1} R_{\geq}^{n} \cap \ldots \cap P_{1} R_{\geq}^{n}\right) \leq \operatorname{card} R^{n}=c$.

So
$\operatorname{card}\left(P_{0} R_{\geq}^{n} \cup P_{1} S_{\geq}^{n-1} \cup \ldots \cup P_{n !} R_{\geq}^{n}\right)+\operatorname{card}\left(P_{0} R_{\geq}^{n} \cap P_{1} R_{\geq}^{n} \cap \ldots \cap P_{n !} R_{\geq}^{n}\right)=c$ $\operatorname{ard}\left(R^{n}\right)+k=c+k=c$.

Therefore $\operatorname{card}\left(R_{\geq}^{n}\right)=\operatorname{card}\left(R^{n}\right)$. That is $R_{\geq}^{n}$ bijective with $R^{n}$.

we have:

Corollary 2.3: Let

$R_{\geq}^{n}=\left\{x \in R^{n}: x_{1} \geq \ldots \geq x_{n}\right\} \subseteq R^{n}$.

Then $R_{\geq}^{n}$ admits a differentiable structure with dimension $n$.

Proof 2.4: Since $R^{n}$ is a differentiable manifold, Corollary follows from the above Lemma and Remark 2.1, part 1.

We denote bijection in lemma 2.1 by $T: R_{\geq}^{n} \rightarrow R^{n} . T$ is a diffeomorphism and therefore if $M$ is a submanifold of $R^{n}$, then $T^{1}$ $(M)$ is a submanifold of $R_{\geq}^{n}$.

we define

$\operatorname{diag}\left[R_{z}^{n}\right]=\left\{\operatorname{diag}[r]=\operatorname{diag}\left[r_{1}, \ldots, r_{n}\right] \mid\left(r_{1}, \ldots, r_{n}\right) \in R_{z}^{n}\right\} \subseteq S(n, R)$.

(diag [] is a matrix with nonzero elements only in main diagonal.) Thus $\operatorname{diag}\left[R_{z}^{n}\right]$ is a differentiable manifold with dimension $n$.

Proposition 2.5: The eigenvalue function $\lambda$ is a differentiable function.

Proof 2.6: The orthogonal Lie group $O(n, R)$ acts as a Lie transformation group on $S(n, R)$ as following

$\Phi: O(n, R) \times S(n, R) \rightarrow S(n, R)$

$(P, A) \rightarrow P A P^{t}$

Where $P^{t}$ is the transpose of matrix $P$.

So if $P \in O(n, R)$, the function $\Phi_{p}: S(n, R) \rightarrow S(n, R)$ defined by $A \rightarrow \Phi(P, A)$ is a diffeomorphism of $S(n, R)$ onto itself and so it is a submersion. This follows that the function $T: O(n, R) \times \operatorname{diag}\left[R_{z}^{n}\right] \rightarrow S(n, R)$ defined by

$(P, \operatorname{diag}[s]) \rightarrow P \operatorname{diag}[s] P^{t}$

is a submersion. Let $\rho$ be projection function on second factor

$\rho: O(n, R) \times R^{n} \rightarrow S(n, R)$

$(P, \operatorname{diag}[s]) \rightarrow \operatorname{diag}[s]$,

then $\rho$ is differentiable and $\rho=\lambda_{\circ} T$. Hence, by the Proposition 1.1, part $1, \lambda$ is differentiable.

Proposition 2.5 has some important results about the properties of $\lambda$ :

\section{Corollary 2.7}

1. $\lambda$ is a submersion.

2. Let $A \in S(n, R)$ with spectral decomposition $A=P \operatorname{diag}\left[\lambda_{1}, \ldots, \lambda_{n}\right]$ $P^{t}$. The Jacobian of $\lambda, \nabla \lambda$, is given by

$\nabla \lambda_{A} h=P h P^{t}$,

Where $h \in S(n, R)$

3. $\lambda$ is an open mapping.

\section{Proof 2.8}

1. Let $A \in S(n, R)$ and $A=P \operatorname{diag}[\lambda] P^{t}$ its spectral decomposition. If $\varphi$ be Lie transformation group on Proposition 2.5, then 


\section{$\phi_{P}: \operatorname{diag}\left[R_{z}^{n}\right] \rightarrow S(n, R)$}

defined by $\varphi_{p}(\operatorname{diag}[s])=P \operatorname{diag}[s] P^{t}$ is a differentiable section of $\lambda$. Hence, the state follows from Proposition 1.1, part 2.

2. It is well known that: If $A$ is any point in the domain of a submersion $\lambda: S(n, R) \rightarrow R^{n}$ there exist charts $x, y$ of $S(n, R) \rightarrow R^{n}$ at $A, \lambda(A)$ respectively such that the function $y_{\circ} \lambda_{\circ} x^{-1}$ is $(z, w) \rightarrow z$.It is clear that $y$ is identity function on $R^{n}$ and $x^{-1}$ is a $P \in O(n, R)$ such that $A=P \lambda(A) P^{t}$. We can write $\lambda$ such that, on some neighborhood of $A \in S(n, R)(P, \operatorname{diag}[s]) \rightarrow \operatorname{diag}[s]$. In other words $P A P^{t}=\operatorname{diag}[s]=\lambda(A)$. By multiplying both sides of this equality by $P^{t}, P$ from the right and left, respectively, we have

$P^{t} \lambda(A) P=A(1.1)$

Suppose that $A$ is fixed and $h$ belong to a small neighborhood of $A$.With differentiating from both sides of (1.1) we have $P^{t} \nabla \lambda_{A} P=I$, where $I$ is identity matrix in $S(n, R)$. Therefore $P^{t} \nabla \lambda_{A} P=h$. This yields $\nabla \lambda_{A} P=P h P^{t}$

3. Any submersion is an open mapping.

Example 2.1: The set $M$ of all real symmetric matrices of order $2 \times 2$ with distinct eigenvalues is an open subset of $S(2, R)$.So, by the above Corollary, $\lambda(M)$ is an open subset of $R_{z}^{2}$.

\section{More Spectral Manifolds (Isotropic)}

For fixed subset $M \subseteq R^{n}$, some properties on $M$ remain true on the corresponding set $\lambda^{-1}(M)$ (spectral set). If $M$ is differentiable manifold of $R^{n}$, then $\lambda^{-1}(M)$ will be called spectral manifold. The spectral manifolds are entirely defined by their eigenvalues [10].

For example, if the set $M$ is symmetric, then properties such as closedness, convexity, prox-regular are transferred between $M$ and $\lambda^{-1}(M)[11,12]$.

he set $\lambda^{-1}(M)$ often appears in engineering sciences, often as constraints in feasibility problems (for example, in the design of tight frames in image processing or in the design of low-rank controller in control) $[13,14]$

Also,transfer of differentiable structure of a submanifold $M$ of $R^{n}$ has been studied. We speculate that most of these results do't depend on the property of symmetry. We must rewrite some of these results in the other work. In this section, we study fibers and orbits of $\lambda$ as two types of spectral manifolds.

$\lambda$ determines an equivalence relation $\rho$ on $S(n, R)$, defined by $(A, B) \in$ $\rho$ if and only if $\lambda(A)=\lambda(B)$. The equivalence classes of $\rho$ are similar matrices. In fact, the following Lemma holds for any submersion .

Lemma 3.1: There is an equivalence relation $\rho$ on $S(n, R)$ such that $\frac{S(n, R)}{\rho}$ has a differentiable structure and it becomes a quotient manifold diffeomorphic to $R_{z}^{n}$.

This lemma has an important result about spectral manifolds. Let $L$ be the diffeomorphism in the lemma and $M \subseteq R^{n}$ be a submanifold. Then $L^{-1}(M)$ is a submanifold of $\frac{S(n, R)}{\rho}$.

That is:

Corollary 3.2: Any spectral submanifod in $S(n, R)$ is union of similar matrices in $S(n, R)$.

Similar matrices in $S(n, R)$ have regular submanifold structure:
Lemma 3.3: For $s \in R^{n}, \lambda^{-1}(s)$ (fiber of $\lambda$ )has the structure of a regular submanifold of $S(n, R)$ of dimension $\frac{n(n-1)}{2}$.

Proof 3.4 Since $\lambda$ is a submersion, Lemma follows from Proposition 1.4 .

Let $\varphi$ given by Proposition 2.5. This Lie transformation group sets up an equivalence relation on $S(n, R)$. The equivalence class containing a point $A$ is the range of the function $\varphi A: O(n, R) S(n, R)$ (orbit of $A$ ) and

$\lambda^{-1}(\lambda(A))=$ orbit of $A=\varphi_{A}(O(n, R))$.

The set $H_{A}=\left(\phi_{A}^{-1}\right)(A)$ is called stabilizer of $A$ (or isotropy group). It is well known that if $H_{A}$ is not an open subgroup of $O(n, R)$, then the orbit of $A$ can be given the structure of a regular submanifold of $S(n, R)$ diffeomorphic to the quotient manifold $\frac{O(n, R)}{H_{A}}$. Thus, by the following Lemma, $\lambda^{-1}(\lambda(A))$ is a regular submanifold of $S(n, R)$ of dimension

$\operatorname{dim} \varphi_{A}=\operatorname{dim} O(n, R)-\operatorname{dim} H_{A}$.

Lemma 3.5: For $A \in S(n, R)$, the stabilizer of $A$ is not an open subset of $O(n, R)$.

Proof 3.6: Let $H_{A}$ be an open subgroup of $O(n, R)$. Then the quotient set topology on $\frac{O(n, R)}{H_{A}}$ is discrete. Therefore $\lambda^{-1}(\lambda(A))$ is discrete. This contradicts with Lemma 3.2.

\section{Spectral Functions}

Let $A=P \operatorname{diag}\left[\lambda_{1}, \ldots, \lambda_{n}\right] P^{t}$, for some $P \in O(n, R)$. In, Chen, Qi, and Tseng showed that for any function $f: R \rightarrow R$, one can define a spectral function $f^{\vartheta}: S(n, R) \rightarrow S(n, R)[1,2,15]$ by

$$
f^{0}(A):=P \operatorname{diag}\left[f\left(\lambda_{1}\right), \ldots, f\left(\lambda_{n}\right)\right] P^{t}, A \in S(n, R),(1.2)
$$

which are constant on the orbit of $A$ and the properties of continuity, directional differentiability, differentiability, and continuous differentiability are inherited by $f^{\ominus}$ from $f$. As we shall see, $f$ does not play a large role.

In this section we examine only differentiability and continuous properties by using Lie group tools

Proposition 4.1: Let $A \in S(n, R)$ and $M$ be the orbit of $A$. Then differentiability of $f^{\natural} \mid M$ not depends on the differentiability of $f$.

Proof 4.2: For each $B \in M, \lambda(A)=\lambda(B)$. Let $f_{\circ} \lambda_{i}(B)=f\left(\lambda_{i}(B)\right)=c_{i}, i=, \ldots$ ,n. If $M^{\prime}=\left\{B \in S(n, R) \mid B=\operatorname{Pdiag}\left[c_{1}, \ldots, c_{n}\right] P^{t}\right\}$ be the orbit of $C=\operatorname{diag}\left[c_{1}, \ldots\right.$ ,$c_{n}$ ] then the function $f^{\vartheta}: M \rightarrow M^{\prime}$ induced by $f^{\vartheta}$ is defined

\section{$B \rightarrow \varphi(P, C), \forall P \in O(n, R)$}

where $\varphi$ is Lie transformation action on the Proposition 2.5. Therefore $f^{\vartheta}$ is differentiable.

Proposition 4.3: For function $f: R \rightarrow R$, the following results hold:

1. $f^{\vartheta}$ is differentiable at $A \in S(n, R)$ with eigenvalues $\lambda_{1}, \ldots, \lambda_{n}$ if and only if $f$ is differentiable at $\lambda_{1}, \ldots, \lambda_{n}$.

2. . $f^{\vartheta}$ is continuous at $A \in S(n, R)$ with eigenvalues $\lambda_{1}, \ldots, \lambda_{n}$ if and only if $f$ is continuous at $\lambda_{1}, \ldots, \lambda_{n}$

\section{Proof 4.4}

1. According to the second part of the Corollary 2.7, there exists an open neighborhood $U$ of $A \in S(n . R)$ and open neighborhood $V$ of $\lambda(A)$ in $R_{z}^{n}$ such that $\lambda(U)=V$ and $\lambda$ in these local coordinates is the standard projection 
$\lambda\left(x_{11}, \ldots, x_{1 n}, \ldots, x_{n n}\right)=\left(x_{11}, \ldots, x_{1 n}\right)$.

Therefore we can write

$$
\left.f^{0}(A)=f^{0}\left(x_{11}, \ldots, x_{1 n}, \ldots, x_{n n}\right)=\operatorname{diag}\left[f\left(x_{11}\right), \ldots,\left(x_{1 n}\right)\right)\right]
$$

That is $f^{\vartheta}$ differentiable if and only if $f$ differentiable.

2. This part is a consequence of part 1 .

In the following proposition we have removed symmetric condition and other extra conditions $[16,17]$.

Proposition 4.5: Suppose $f: R^{n} \rightarrow R$ be a function. Then the following assertions are true:

1. $f$ is continuous at $\lambda \in R^{n}$ if and only if $f$ is continuous at $P \lambda$ for any $P \in O(n, R)$.

2. $f$ is differentiable at $\lambda \in R^{n}$ if and only if $f$ is differentiable $P \lambda$ for any $P \in O(n, R)$.

\section{Proof 4.6}

1. $O(n, R)$ acts on $R^{n}$ as a transitively Lie transformation group(with matrix notation). Then $\Phi_{P}: R^{n} \rightarrow R^{n}$ by

$\Phi_{P}(\lambda)=P \lambda$

is a diffeomorphism. But $f(\lambda)=f_{\mathrm{o}} \Phi_{P}\left(P^{t} \lambda\right)$. Therefore two side of state are clear.

2. Proof of this part is similar to part (1). with

In the sequel, corresponding with (1.1), for each function $f: R^{n} \rightarrow R^{n}$

$$
\begin{aligned}
& f(x)=\left(f_{1}(x), \ldots, f_{n}(x)\right)^{t}\left(x \in R^{n}\right), \\
& \text { we define } \tilde{f}(A): S(n, R) \rightarrow S(n, R) \text { by } \\
& \tilde{f}=\operatorname{Pdiag}\left[\left(f_{1} \circ \lambda\right)(A), \ldots,\left(f_{n} \circ \lambda\right)(A)\right] P^{t},(A \in S(n, R))
\end{aligned}
$$

and similar to the Proposition4.3 we can show that if $f: R^{n} \rightarrow R^{n}$ has the property of continuity(respectively, differentiability), then so does the symmetric-matrix function $\tilde{f}$ is defined by (1.3).

Note that $\tilde{f}$ is independent of choice of $P \in O(n, R)$ and that $\tilde{f}(A)$ belongs to $S(n, R)$, [18].

Proposition 4.7: Let $f: R^{n} \rightarrow R^{n}$ be a function with $f(x)=\left(f_{1}, \ldots, f_{n}(x)\right)$ ${ }^{t}\left(a \in R^{n}\right)$, and let $\tilde{f}$ be defined by (1.3). Let $A \in S(n, R)$ with eigenvalue $\lambda_{1}(A), \ldots, \lambda_{n}(A)$ and $\lambda(A)=\left(\lambda_{1}(A), \ldots, \lambda_{n}(A)\right)^{t}$. Then the following assertions are equivalent:

\section{1. $f$ is continuous at $\lambda(A)$}

2. $f$ is continuous at $A$.

Proposition 4.8: Let $f: R^{n} \rightarrow R^{n}$ be a function with $f(x)=\left(f_{1}, \ldots, f_{n}(x)\right)$ ${ }^{t}\left(a \in R^{n}\right)$. Let $A \in S(n, R)$ with eigenvalues $\lambda(A)=\left(\lambda_{1}(A), \ldots, \lambda_{n}(A)\right)^{t}$ and $\lambda(A)=\left(\lambda_{1}(A), \ldots, \lambda_{n}(A)\right)^{t}$. Then the following assertions are equivalent:
1. $f$ is differentiable at $\lambda(A)$
2. $f$ is differentiable at $A$.

\section{Acknowledgments}

The research was supported by Marand Branch,Islamic Azad University.

\section{References}

1. Bahatia R (2012) Matrix analysis. Springer-Verlag.

2. Horn RA, Johnson CR (1985) Matrix analysis. (2ndedn) Cambrige University Press, Cambridge.

3. Kanzow C, Nagel C (2002) Semidefinite programs:new search direction smoothing-type methods and numerical results. SIAM J Optimiz 13: 1-23.

4. Lewis AS, Overton ML (1996) Eigenvalue optimization. In Acta Numerica. Acta Number 5. Cambridge University Press, pp: 149-190.

5. Kemble EC (1958) The fundamental principles of quantum mechanics.Dover New York.

6. Schiff LI (1955) Quantum Mechanics. McGraw-Hill, New York

7. Lewis AS (1996) Convex analysis on the Hermitian matrices. SIAM J. Optimiz 6: 164-177.

8. Brickell F, Clark RS (1970) An introduction to differentiable manifolds. van nostrand reinhold company Ltd.

9. Feng $Y$, Lin ST Set theory and its application. Mariner pub. co.

10. Daniilidis A, Lewis AS, Malick J, Sendov H (2008) Prox-regularity of spectral functions and spectral sets. J. Convex Anal 15: 547-560.

11. Daniilidis A, Malick J, Sendov H (2014) Spectral (isotropic) manifolds and their dimension. hal-0097221, version 1-3.

12. Lewis AS (1999) Nonsmooth analysis of eigenvalues. Math Programming 84 1-24.

13. Tropp JA, Dhillon IS, Heath RW, Strohmer T (2005) IEEE Trans. on Inf. Theory 51: 188-209.

14. Orsi R, Helmke U, Moore JB (2006) A Newton-like method for solving rank constrained linear matrix inequalities. Automatica 42: 1875-1882.

15. Chen X, Qi HD, Tseng P (2003) Analysis of nonsmooth symmetric-matrixvalued functions with applications to semidefinite complementarity problems. SIAM J. Optimiz 13: 960-985.

16. Lewis AS, Sendov HS (2001) Twice differentiable spectral functions. SIAM J Matrix Anal Appl 23: 368-386.

17. Qi HD, Yang X (2004) Semismoothness of spectral functions. SIAM J Matrix Anal 25: 766-783.

18. Mohebi H, Salemi A (2007) Analysis of symmetric matrix valued functions Num. Functional Analysis and Optimization 28: 691-715. 